\title{
CONSÉQUENCES ÉCOLOGIQUES DES INTRODUCTIONS DANS LES HYDROSYSTÈMES : ESSAI DE SYNTHĖSE.
}

\author{
J. HAURY (1), E. PATTEE (2).
}

(1) École Nationale Supérieure Agronomique de Rennes - Département E.E.R.N., Lab. Écologie et Sciences Phytosanitaires ; Institut National de la Recherche Agronomique, Lab. Écologie Aquatique, 65 rue de Saint-Brieuc, 35042 Rennes Cedex, France.

(2) Université Claude Bernard Lyon-I, ESA CNRS Écologie des Eaux Douces et des Grands Fleuves, 43 boulevard du 11 novembre 1918, 69622 Villeurbanne Cedex, France.

\section{RÉSUMÉ}

Un essai de bilan des effets écologiques des introductions d'espèces est présenté à partir des exposés des orateurs de ce colloque et de la bibliographie. L'aspect inéluctable de la dispersion des espèces au cours du temps et l'efficacité toute relative des barrières intracontinentales qui s'y opposent sont soulignés. Les mécanismes en cause - vagilité et fécondité des espèces introduites, efficacité des barrières, structure des réseaux trophiques récepteurs, degré de saturation de leurs niches écologiques - sont étudiés en regard du fonctionnement des hydrosystèmes, montrant la complexité du problème. Des effets écologiques très variés sont décrits, dont beaucoup sont non intentionnels et inattendus; un exemple bibliographique est développé, mettant en évidence la diversité des effets de la même introduction selon le milieu récepteur. II en ressort la nécessité préliminaire absolue d'examiner à la fois la biologie des espèces à introduire et le fonctionnement de l'écosystème récepteur. Un principe de précaution est préconisé face à toute introduction volontaire, et une réglementation sévère et efficace face aux introductions involontaires. Le besoin d'une permanence d'agents de terrain pour établir une veille écologique est souligné. Face au manque de données objectives, des recherches scientifiques sur les effets écologiques des introductions passées ou possibles doivent être initiées ou poursuivies dans un cadre écosystémique, impliquant au minimum un suivi des écosystèmes risquant d'être colonisés.

Mots-clés : introductions, hydrosystèmes, dispersion, effets écologiques, théorie écologique.

\section{ECOLOGICAL EFFECTS OF SPECIES INTRODUCTIONS IN HYDROSYSTEMS : AN ATTEMPT OF EVALUATION.}

\begin{abstract}
The present attempt at evaluating the ecological impacts of species introductions is based upon the communications given at this meeting and the literature. The inescapable character of species dispersion over time and the low efficiency of within-continent barriers are stressed. The mechanisms implied - mobility and fecundity of the introduced species, efficiency of the dispersion-limiting barriers, structure of the recipient trophic networks and degree of saturation of their ecological niches - are detailed in the context of hydrosystem functioning. They reveal the complexity of the problem. Introductions have produced quite diverse ecological effects, many of them unwanted and/or unexpected. An example from the literature shows that the same introduction has had different consequences according to the environment. Before any introduction, it is therefore necessary to study both the biology of the species to be introduced
\end{abstract}


and the functioning of the receiving ecosystem. Great wariness is recommended before any wilful introduction. Severe and efficient regulation must be applied against unintentional introductions. The need for regular field monitoring by permanent ecological personnel is stressed. Considering the lack of proper informative data, scientific investigations on the ecological effects of past or possible introductions must be undertaken or continued, at least on the ecosystems exposed to introductions.

Key-words : introductions, freshwater ecosystems, dispersion, ecological effects, theoretical ecology.

\section{INTRODUCTION}

La question des introductions pose, de façon immédiate, celle de leurs répercussions écologiques. Ce sont d'ailleurs ces impacts qui justifient le " principe de précaution " retenu dans la loi, et qui a été évoqué à plusieurs reprises (BENTATA, 1997 ; GUEVEL, 1997). Au terme de cette manifestation, un bilan sur les effets écologiques des introductions s'avère nécessaire, partant des expériences exposées pour arriver à une vision plus générale, en fonction des concepts de l'écologie fonctionnelle. Par ailleurs, dans un contexte de préservation de la biodiversité, les relations entre ces introductions et la richesse spécifique doivent être élucidées.

Dans cet article de bilan, le choix a été fait d'exploiter les présentations des différents orateurs, tout en recourant aussi à des éléments bibliographiques extérieurs. Un examen des mécanismes écologiques permettra, ultérieurement, de comprendre les effets décrits et de proposer une réflexion préliminaire à toute introduction d'espèce.

\section{ÉVIDENCES PRÉALABLES ET DÉFINITIONS}

\section{Des barrières naturelles à franchir}

Le milieu des eaux continentales (eaux douces, en majorité) est, par définition, circonscrit par le milieu terrestre qui le divise en bassins distincts, lesquels (du fait de l'écoulement de ces eaux) aboutissent indépendamment à la mer : ce sont des "îles continentales " (BARBAULT, 1992), selon la théorie biogéographique des îles de MACARTHUR et WILSON (1967). En raison de leur habitat fragmenté, plusieurs espèces aquatiques forment des métapopulations. La colonisation d'un bassin à partir d'un autre implique donc le franchissement de barrières terrestres ou marines. Les possibilités de franchissement de ces barrières sont inversement proportionnelles à la distance à parcourir, tout en dépendant des organismes et de leur inféodation plus ou moins stricte à l'eau. Ainsi, les barrières océaniques et désertiques sont les plus efficaces (SASTRE, 1997 ; DAVAINE et BEALL, 1997). De plus, les eaux continentales se subdivisent en eaux douces ou saumâtres, eaux courantes rapides ou lentes, eaux stagnantes, eaux souterraines, situées à des altitudes et sous des latitudes différentes qui font que n'importe quel organisme aquatique ne peut pas vivre dans, voire migrer à travers, n'importe laquelle de ces eaux. Si les modalités de franchissement naturel des barrières ne sont pas encore parfaitement élucidées, notamment pour les organismes strictement aquatiques les plus gros tels les poissons (transport par les oiseaux, par les tornades, capture de rivières les unes par les autres aux époques géologiques, ayant par exemple permis le passage du Danube au Rhin, puis du Rhin au Rhône à l'ère tertiaire (PERSAT et KEITH, 1997), on observe que, tout en étant parfaitement isolés les uns des autres, des bassins voisins ont des faunes identiques ou très voisines. Le franchissement naturel des barrières terrestres a donc eu lieu de tout temps. II se poursuit de nos jours, avec par exemple un enrichissement de la faune aquatique de l'Europe à partir de l'est. En effet, une grande partie de la faune aquatique tertiaire a été éliminée par les glaciations (PERSAT et KEITH, 1997) et les limites que constituent la Méditerranée au sud et les Pyrénées au sud-ouest sont difficilement franchissables. Par rapport aux régions plus continentales, il en résulte une " insaturation des communautés pisciaires " dans les hydrosystèmes français, notamment les lacs d'altitude (DELACOSTE et al., 1997) ; ce que l'on retrouve avec les Azolla qui n'ont regagné le territoire français qu'au XIXème siècle (DUTARTRE et al., 1997). II n'en est pas moins vrai que les populations des eaux continentales ont (sauf 
dévalaison dans un hydrosystème donné) des pouvoirs de dissémination moindres que celles du milieu terrestre et, a fortiori, du milieu marin ; elles se prêtent, par conséquent, plus facilement à des interventions de gestion.

\section{Gérer les propagations naturelles et les introductions ?}

Comment, et à quel titre, interdire cette propagation naturelle, ne serait-ce que le passage des espèces aquatiques étrangères par les canaux qui relient maintenant les bassins fluviaux entre eux? Les introductions accidentelles ou volontaires, thème de ce séminaire, sont par définition le fait de l'homme. Elles ne représentent que l'une des modalités du franchissement des barrières terrestres et océaniques et de ce repeuplement de l'Europe Occidentale, le franchissement étant quasi inéluctable à l'échelle géologique.

Ces introductions ont existé depuis l'apparition de l'homme (SASTRE, 1997), et notre vie actuelle serait difficilement concevable sans elles : que seraient une Europe sans pomme de terre, maïs, tabac, tomate, une Amérique sans cheval, une Australie sans mammifères euthériens, une Nouvelle-Zélande sans hyménoptères butineurs? Notamment pour les poissons, on recense désormais une forte proportion d'espèces introduites dans la plupart des hydrosystèmes, tant français (KEITH et ALLARDI, 1997) qu'étrangers (SPENCER et al., 1991; CRIVELLI, 1995 ; ELVIRA, 1995). Le même phénomène est observé pour les macrophytes riverains de l'Adour (PLANTY-TABACCHI, 1997). Ainsi, en eaux douces, on attribue aux Romains l'extension de la carpe (Cyprinus carpio) (COWX, 1997). Désormais, cette espèce, originaire d'Asie centrale, est devenue cosmopolite, suite aux introductions successives et à leurs conséquences (ALLAN, 1995). II n'empêche que notre histoire récente comporte des exemples d'introductions dont l'homme se serait bien passé, telles celles du poisson-chat et de la perche-soleil dans nos eaux françaises.

\section{La nature et les causes des introductions}

Elles peuvent avoir été faites dans un but avoué d'acclimatation ou de naturalisation:

- en vue d'une production végétale (essais d'utilisation de la jacinthe d'eau en épuration - DUTARTRE et al., 1997) ou animale accrue, soit par introduction directe de l'organisme recherché (salmonidés dans les eaux de haute altitude ou forte latitude - DELACOSTE et al., 1997 ; ROCHE et MATTEI, 1997 ; DAVAINE et BEALL, 1997), soit par enrichissement d'un échelon trophique dans l'espoir d'accroître la production d'un autre échelon trophique (crevette Mysis relicta dans les lacs du Nord de l'Amérique pour accroitre la production de truites - NORTHCOTE, 1972) ;

- dans des buts plus spécifiques tels que la pêche sportive (truite arc-en-ciel dans le vallon d'Estibère : DELACOSTE et al., 1997), la production de cuisses de grenouilles (NEVEU, 1997), le remplacement des espèces d'écrevisses malades de la " peste " (LAURENT, 1997), la lutte biologique contre les moustiques (Gambusie - ROSECCHI et al., 1997), la constitution d'habitats favorables pour la fraie des poissons phytophiles (introduction de Myriophyllum brasiliense dans des étangs de pêche).

Elles peuvent aussi être seulement accidentelles : évasion d'individus provenant d'élevages (COWX, 1997 ; MAURIN, 1997), de jardins botaniques ou d'agrément comme les introductions de Elodea canadensis dans le Limousin et de Salvinia molesta en Asie, via les jardins botaniques européens (COOK et GUT, 1971, in COOK, 1985), voire de plans d'eau ou de berges où ils étaient introduits à des fins ornementales (Mimulus guttatus sur les rives des cours d'eau du Nord, Jussie - DUTARTRE et al., 1997), déversement d'aquariums ou rejet d'individus par leurs propriétaires afin de s'en défaire (tortues de Floride acclimatées dans de nombreux plans d'eau - SERVAN et ARVY, 1997 ; MINISTĖRE DE L'ENVIRONNEMENT, 1995). Les coques des bateaux (éventuellement transportés par voie de terre) ou les lests constituent aussi un moyen de dispersion, notamment pour les organismes fixés et peu mobiles par ailleurs (c'est, par exemple, le mode de colonisation probable initial de la composée Cotula coronopifolia en Bretagne, ou des Paspalum des rives de la Basse-Loire, et de Dreissena polymorpha dans le lac Léman (MATTHEY, 1966). 
Aussi, à partir de l'exemple des introductions passées, de ce que l'on peut percevoir de leurs conséquences écologiques et des modalités de leur gestion, il est nécessaire de regarder vers l'avenir où restent certains choix à l'homme : introductions à favoriser, introductions à éviter, d'après quels critères, et comment en décider? Tel était le débat de fond de ce colloque, envisagé à travers toute une série d'exemples concrets. Enfin, se pose aussi la question de la lutte contre les nuisances entraînées par certaines espèces introduites.

\section{CADRE FONCTIONNEL POUR ANALYSER LES EFFETS ÉCOLOGIQUES DES INTRODUCTIONS}

Deux aspects sont donc à envisager : I'hydrosystème récepteur et la population introduite.

Les hydrosystèmes continentaux ont des particularités qui ont été synthétisées dans deux ouvrages récents sur les eaux courantes (AMOROS et PETTS, 1993) et stagnantes (POURRIOT et MEYBECK, 1995). La plupart sont continus de la source à la mer, si bien qu'une introduction dans un point d'une rivière, et même d'un plan d'eau, aura toutes chances de concerner à terme l'ensemble du réseau. Leur richesse spécifique dépend de l'équilibre entre les arrivées naturelles ou anthropiques (introductions) et les extinctions d'espèces qui se produisent sans arrêt (MACARTHUR et WILSON, 1967 ; HUTCHINSON, 1975).

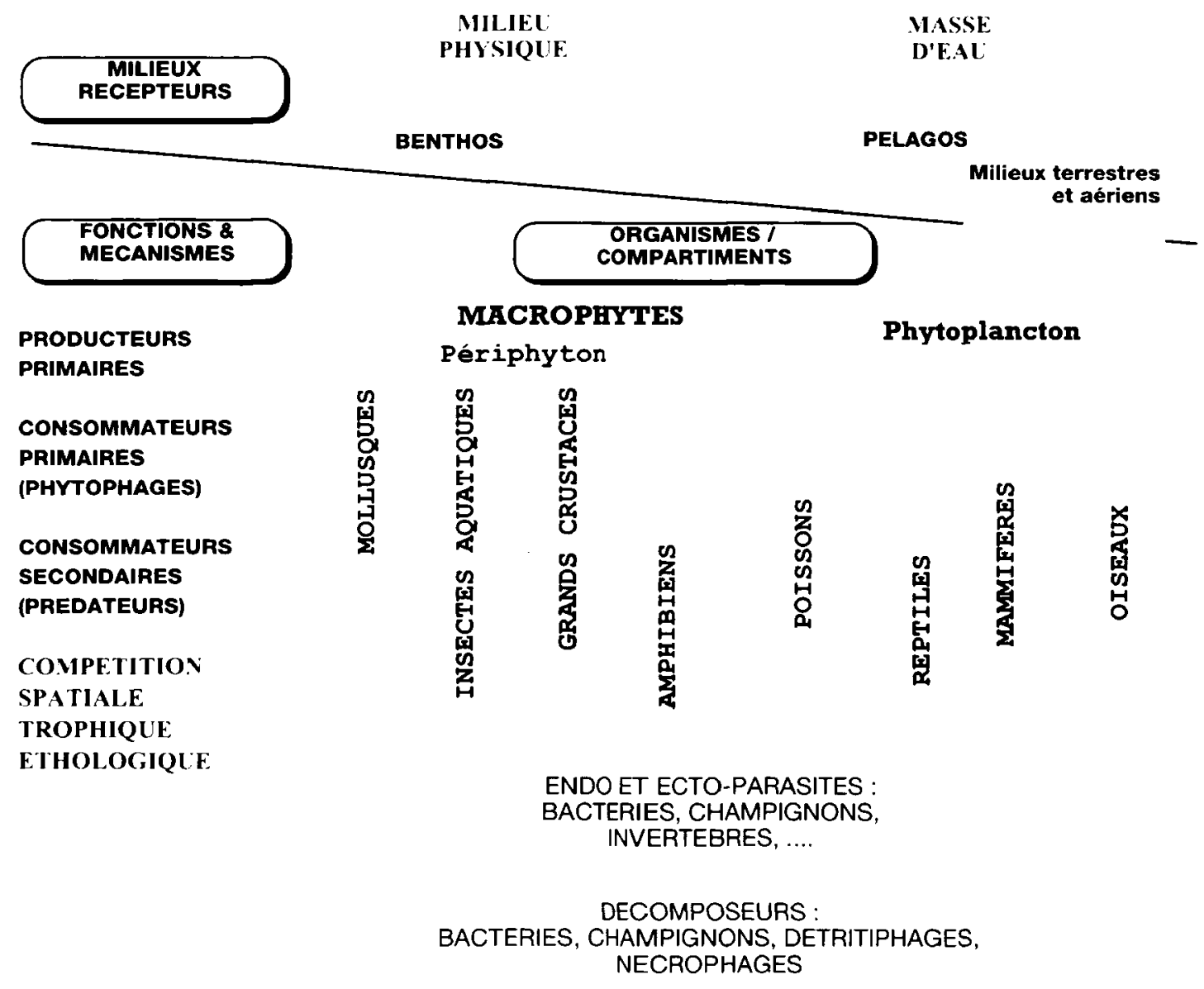

Figure 1

Analyse multicompartiments des hydrosystèmes et groupes d'espèces introduites.

Figure 1

Multicompartment analysis of hydrosystems and groups of introduced species. 
L'analyse de l'état de leurs compartiments (figure 1) correspond à la description du milieu physique solide, de la masse d'eau, des producteurs primaires benthiques ou pélagiques, microphytes et macrophytes, des consommateurs, qu'ils soient herbivores ou prédateurs, mais aussi des décomposeurs, que tous ces organismes soient étudiés par groupe taxonomique ou par échelon trophique. Le groupe fonctionnel correspond à l'ensemble des espèces situées à une même place dans les réseaux trophiques, et qui se partagent alors certaines dimensions d'une même niche écologique. Malheureusement, certains compartiments sont assez mal connus, ou insuffisamment inventoriés, si bien qu'il est difficile de faire le point sur les introductions d'espèces ; c'est par exemple le cas pour les invertébrés, et spécialement les insectes aquatiques (ALLAN, 1995), et pour les diatomées, où des espèces d'origine étrangère sont assez fréquemment rencontrées, si bien que les clés de détermination deviennent rapidement incomplètes (COSTE, 1994). Ces compartiments sont en interrelations fonctionnelles et il existe, bien évidemment, des équilibres au sein de chaque compartiment. On peut donc émettre l'hypothèse de travail qu'à partir d'un écosystème " en équilibre ", les introductions vont modifier certains aspects du fonctionnement, notamment les chaînes et réseaux trophiques. Par ailleurs, la perception des équilibres écologiques est éminemment anthropique, et la gravité de nombreux effets écologiques est réinterprétée (voire même mesurée, et donc connue) en fonction des nuisances perçues par l'homme (par exemple, l'entrave à la navigation, la régression du poisson "pêchable "). Comme nous ne savons pas exactement ce qu'est un système " en équilibre " ou " en bonne santé " (LEVEQUE, 1994), cet équilibre étant au mieux métastable, ni toujours la représentativité des données acquises, les éléments fournis par la communauté scientifique seront plus des indications que des certitudes d'effets. Tous les systèmes ne présentent pas un même risque "d'invasibilité ", comme le signalent certains auteurs (BURKE et GRIME, 1996, par exemple) ; ce qui suppose de comprendre les mécanismes qui régissent cette sensibilité à l'“ invasion ". Si l'usage a consacré le terme d'invasion, associé aux idées de nuisance et de prolifération (GROVES et BURDON, 1986), ces termes restent fort ambigus et anthropocentriques, et l'emploi du terme d'invasion notamment est déconseillé (SASTRE et BENTATA, 1997).

Les caractéristiques des espèces introduites déterminent la suite de l'introduction. II est donc nécessaire de développer une grille d'analyse de ces caractéristiques biologiques des espèces susceptibles d'avoir des effets écologiques importants et, a contrario, de celles qui n'auront que peu ou pas d'effet (mesurable ou visible) sur l'écosystème récepteur.

\section{BIOLOGIE DES ESPĖCES INTRODUITES ET ENVAHISSANTES}

Trois phases complémentaires sont à envisager pour l'analyse des relations entre la biologie des espèces introduites et leurs effets dans les hydrosystèmes : l'introduction stricto sensu, l'installation de la population, son éventuelle extension. En effet, suite aux introductions, plusieurs avenirs peuvent être décrits schématiquement et ont été analysés par les biogéographes (SASTRE, 1997):

\section{1 - Disparition à terme des espèces introduites.}

2 - Maintien de l'espèce, sans qu'il y ait possibilité de reproduction : c'est l'acclimatation, le maintien de l'espèce dépendant de l'apport de nouveaux individus.

3 - Maintien de l'espèce accompagné de la possibilité de reproduction : ceci correspond à la naturalisation ; le cas particulier de la possibilité de multiplication végétative qui entraine le maintien ou l'extension des populations peut être assimilé, du point de vue écologique, à une naturalisation.

La phase d'introduction dépend des vecteurs et, pour les introductions volontaires, ne dépend pas (ou peu) de la biologie des espèces considérées. En revanche, pour les introductions accidentelles, les relations classiques liées à la dissémination des espèces sont à étudier et correspondent aux mécanismes en jeu dans les phases d'extension. 
L'installation des espèces est le phénomène majeur qui détermine les nuisances liées aux introductions. L'ensemble des traits biologiques correspond à une plus ou moins grande capacité d'installation des espèces (NEWSOME et NOBLE, 1986). Pour les organismes aquatiques, ARTHINGTON et MITCHELL (1986) ont comparé le cas des plantes et des poissons.

- La possibilité de supporter les aléas climatiques correspond à l'amplitude écologique des espèces, mais est aussi favorisée par la forte inertie thermique des hydrosystèmes. A priori, l'introduction dans des zones homologues (comme les lacs alpins, pour les salmonidés sténothermes d'eau froide) diminue la probabilité d'échec de l'opération. Toutefois, lorsqu'il y a introduction dans des zones climatiques différentes de celles de l'aire d'origine, des possibilités d'acclimatation subsistent, surtout lorsqu'il n'y a pas d'écarts climatiques trop importants (hivers trop froids, prise en glace) pendant plusieurs années, ce qui permet l'adaptation progressive des populations, qui supporteront ensuite des conditions plus extrêmes ; ce phénomène est impliqué dans l'installation de la majorité des espèces subtropicales proliférantes, comme le myriophylle du Brésil.

- La capacité adaptative s'accompagne souvent d'une polyploïdie chez les plantes envahissantes (comme la jacinthe d'eau - BARRETT, 1979, in BARRETT et RICHARDSON, 1986), ou Salvinia molesta (pentaploïde stérile selon LOYAL et GREWAL, 1966, in BARRETT et RICHARDSON, 1986), contrairement aux animaux chez lesquels les anomalies génétiques, entraînant souvent une stérilité non compensée par des possibilités de multiplication asexuée. apparaissent comme un facteur défavorable. Cette polyploïdie peut aussi apparaître suite à la colonisation.

- Des dérives génétiques mettant en jeu des traits biologiques polygéniques peuvent constituer une réaction consécutive à l'introduction, comme MURRAY et al. (1986) le citent pour les Gambusies. Toutefois, toute dérive génétique ne présente pas forcément un caractère favorable à l'adaptation. Par ailleurs, des dérives génétiques peuvent aussi exister sur des loci enzymatiques a priori non polygéniques, comme DAVAINE et BEALL (1997) l'ont vérifié par électrophorèse sur des populations génétiquement homogènes de salmonidés introduits dans les lles Kerguelen en isolement reproducteur.

- Les caractéristiques des plantes aquatiques envahissantes sont leur grande amplitude écologique, leur peu d'exigences quant aux conditions de germination, un pouvoir germinatif élevé et une forte longévité des semences, une croissance embryonnaire rapide et une capacité précoce et durable à la reproduction, une forte auto-compatibilité, une forte capacité de bouturage et de multiplication végétative, et surtout un fort pouvoir compétitif grâce à une croissance active.

- Par ailleurs, certaines espèces sont favorisées par l'eutrophisation des eaux qui active leur croissance, comme Myriophyllum heterophyllum (SYTSMA et ANDERSON, 1993) ou comme Salvinia molesta qui utilise l'azote ammoniacal préférentiellement aux autres formes de l'azote (CARY et WEERTS, 1983). Un phénomène comparable de moindre sensibilité envers les dégradations de la qualité de l'eau est fréquemment observé chez les animaux introduits. C'est par exemple le cas d'Orconectes limosus, qui colonise aussi les eaux de seconde catégorie piscicole (VIGNEUX, 1997). De par son régime alimentaire, le hotu est un nettoyeur de périphyton excédentaire (NELVA, 1997).

- De surcroît, l'absence d'ennemis naturels (parasites ou prédateurs) favoriserait les populations introduites par rapport aux espèces indigènes.

- Pour les animaux, la faible spécialisation du régime alimentaire permet une adaptation à des ressources nouvelles. Toutefois, pour les introductions destinées à la lutte biologique, notamment contre les macrophytes, c'est justement cette spécialisation du régime alimentaire qui est recherchée (DUTARTRE et HAURY, communication orale).

- Par ailleurs, des stratégies alimentaires particulières (comme celle du hotu), mais aussi surtout des comportements de reproduction d'espèces qui construisent des nids, gardent leurs œufs et les aèrent (ROSECCHI et al., 1997), s'avèrent des facteurs favorables de compétitivité, notamment en milieu dégradé. 
L'extension des espèces végétales dépend de leur aptitude compétitive, mais aussi des modes de dispersion des diaspores. De façon générale, les espèces à multiplication végétative, comme beaucoup de macrophytes, sont susceptibles de grandes phases d'extension. Pour les animaux, les stratégies adaptatives des espèces introduites correspondent le plus souvent à une stratégie r de forte capacité reproductrice et, souvent, à la capacité de coloniser des milieux neufs ou des niches inoccupées.

De façon générale, dans la dynamique des populations introduites, on observe assez généralement une phase de prolifération suivie d'une certaine stabilisation, voire d'une disparition. L'éradication pendant cette phase de pullulation est, en général, impossible (les quelques cas d'éradication réussie correspondant à des populations restées localisées, comme le castor américain dans I'Yonne - MAURIN, 1997).

\section{LES EFFETS INDUITS}

Les effets les plus variés ont été décrits :

1 - Aucun effet apparent négatif jusqu'à présent, soit que l'espèce introduite ne s'installe pas (échecs d'introduction) ou finit par disparaître, soit qu'elle s'insère entre les espèces présentes sans répercussions sur elles, ce qui correspond apparemment à un gain de biodiversité (Mimulus guttatus, Hibiscus roseus, I'hépatique Dumortiera hirsuta - DUMAS et HAURY, 1995 ; certains amphibiens introduits - NEVEU, 1997, mais aussi, semble-t-il, certains des poissons introduits récemment en Camargue, notamment le poisson-chat et la perche-soleil, dont les niches alimentaires ne recouvrent aucune de celles des autres espèces autochtones de ces milieux), soit qu'elle reste dominée par les espèces autochtones, comme les salmonidés introduits dans les ruisseaux pyrénéens d'altitude en sympatrie avec la truite fario (DELACOSTE et al., 1997).

2 - Modification des biotopes. Ces effets peuvent être négatifs : percement des digues par le rat musqué, obstruction de canalisations par les petits bivalves récemment parvenus en France, ou l'amphipode Corophium (KHALANSKI, 1997), obstruction de rivières par la végétation d'espèces introduites (Egeria densa), ou comblement partiel ou accéléré d'étangs (PIPET et al., communication orale). Ces modifications peuvent aussi correspondre à la création de milieux favorables, au moins temporairement, pour la fraie des poissons phytophiles par exemple, mais aussi pour certains parasites ou vecteurs de parasites.

3 - Transport de parasites (BLANC, 1997), y compris bouclage du cycle larvaire (par la dresseine, dans le cas de la bucéphalose larvaire).

4 - Apparition de production piscicole là où il n'y en avait pas (lacs de hautes latitudes ou de montagne - DELACOSTE et al., 1997 ; DAVAINE et BEALL, 1997).

5 - Parfois, compétition entre espèces introduites (Dreissena / Corbicula / Corophium en Moselle - BACHMANN et al., 1997 ; Elodea canadensis / Egeria densa - DUTARTRE et HAURY, données non publiées), voire persistance de la seule espèce la plus adaptée au milieu considéré. Cette compétition peut se traduire par une succession dans les espèces introduites, comme c'est sans doute le cas lors du remplacement de Elodea canadensis par $E$. nuttallii dans l'Est de la France (THIEBAUT et al., 1997).

6 - Accroissement (en biomasse ou en valeur marchande) des rendements existants, correspondant à des rapports de productions entre prédateurs et proies (sandre - COWX, 1997 ; grenouille taureau - NEVEU, 1997 ; tilapias et Lates dans le lac Victoria où les introductions ont multiplié la production halieutique par un facteur de 5 - LEVEQUE, 1997). Des réintroductions ou des soutiens de population peuvent avoir le même effet (BAGLINIERE et al., 1990).

7 - Effondrement des effectifs ou de la production des espèces indigènes (voire de la production totale) par compétition (carpe chinoise, grémille) ou prédation (par le sandre, peutêtre par la grenouille taureau - NEVEU, 1997), destruction de la végétation par les carpes chinoises 
ou les rongeurs (COWX, 1997 ; MOUTOU, 1997), extinction (par compétition ou prédation) d'espèces indigènes menacées (érismature à tête blanche gravement concurrencée par l'érismature rousse - PERENNOU, 1997) et leur remplacement par d'autres espèces répandues ailleurs ou sans valeur économique (comme Orconectes limosus - LAURENT, 1997). Cet effet dépressif sur les espèces indigènes, et plus spécialement les endémiques, semble assez général, même si les exemples sont rarement bien documentés (CRIVELLI, 1995) : ainsi, il est observé pour les poissons dans plus des trois quarts des 31 exemples étudiés par ROSS (1991) en Amérique du Nord.

8 - Simplification des réseaux trophiques, souvent ceux à forte biodiversité et fort taux d'endémisme (LEVEQUE, 1997), en particulier lors de l'introduction des super prédateurs, comme le Lates niloticus dans le lac Victoria qui a entraîné la disparition de dizaines de cichlidés endémiques (KAUFMAN, 1992). L'introduction de la crevette opossum, Mysis relicta, pour favoriser la production des salmonidés dans les lacs nord-américains, a également radicalement modifié leurs réseaux trophiques (NORTHCOTE, 1972; RICHARDS et al., 1975 ; SPENCER et al., 1991)

9 - Hybridation avec les espèces ou souches indigènes (NEVEU, 1997 ; PERENNOU, 1997), introgression génique (BERREBI, 1997).

10 - Enfin, la complexité du problème est illustrée par l'exemple de Mysis relicta dans plusieurs lacs nord-américains, exemple que nous avons choisi de développer. Dans les lacs où cette crevette a été introduite, existaient une ou plusieurs populations autochtones de salmonidés. Mais les effets ont été variables.

10.1 - Mysis relicta a été introduite dans le lac Kootenay pour favoriser la production de salmonidés, dans un contexte d'eutrophisation. Des proliférations phytoplanctoniques, au déterminisme non élucidé, ont été observées après l'introduction. Oncorhynchus mykiss (la truite arc-en-ciel) a peu utilisé cette crevette, contrairement à $O$. nerka (le saumon Kokanee). Ces saumons Kokanee, notamment les gros individus, ont changé leur régime alimentaire; il en est résulté un fort taux de croissance et une augmentation des effectifs, si bien que de très gros individus sont désormais pris dans ce lac (NORTHCOTE, 1972). Par ailleurs, toujours selon NORTHCOTE (1972), $M$. relicta pourrait être responsable du déclin des populations de Daphnia et Bosmina.

10.2 - Dans le lac Tahoe, l'introduction de $M$. relicta et de $O$. nerka a entrainé une forte régression des daphnies Daphnia rosea et $D$. pulex et de Bosmina longirostris, ainsi que l'ont observé RICHARDS et al. (1975) : la crevette et le saumon se nourrissent tous deux des cladocères. Mais, dans ce lac profond, la crevette séjourne essentiellement dans les eaux profondes durant le jour, pour monter s'alimenter à partir du zooplancton la nuit : elle est donc peu utilisée par $O$. nerka, qui chasse à vue le jour. En revanche, un changement du régime alimentaire de l'autre salmonidé, Salvelinus namaycush, a été observé : ce dernier a quasiment abandonné les cladocères pour les crevettes.

10.3 - Dans le lac Flathead, plutôt oligotrophe, O. nerka, introduit en 1916, avait progressivement remplacé les salmonidés indigènes, Salvelinus confluentus et Oncorhynchus clarki lewisii (SPENCER et al., 1991). Suite à l'introduction de M. relicta en 1981, ces auteurs ont observé une régression des cladocères, comme dans le lac Tahoe ; ils soulignent, en outre, que les copépodes ont également été affectés. Ils attribuent les proliférations phytoplanctoniques à cette régression du zooplancton, bien que l'eutrophisation en demeure la cause principale. $M$. relicta s'est montrée un compétiteur redoutable pour le saumon Kokanee, dont le déficit dans les affluents où il venait se reproduire a entraîné une régression des aigles, des ours, des visons et des loutres, qui s'en nourrissaient.

Ainsi, malgré une position intermédiaire similaire, la crevette n'a pas produit partout le même effet sur les niveaux trophiques supérieurs. 
La plupart de ces phénomènes peuvent être classés sous la rubrique " déséquilibres biologiques " mentionnés et proscrits par la législation (BENTATA, 1997 ; GUEVEL, 1997), alors que d'autres observateurs considèrent certains de ces changements comme favorables et d'autres comme défavorables. Les effets négatifs sont appelés “Effet Frankenstein " par MOYLE et al. (1986, in SPENCER et al., 1991). La qualification bénéfique ou défavorable des effets et l'estimation de leur ampleur dépendent beaucoup des observateurs. Ces effets n'ont, par ailleurs, pas une signification unique en termes de mécanismes écologiques et donc de possibilités d'action.

\section{GÉNÉRALISATION : LES MÉCANISMES EN CAUSE}

\section{Contexte géographique}

D'un point de vue général et par suite des glaciations mentionnées plus haut, la diversité spécifique, et notamment piscicole, des eaux continentales d'Europe Occidentale est beaucoup plus faible que celle de régions comparables sur d'autres continents (PERSAT et KEITH, 1997). II est clair que les eaux douces européennes sont encore relativement insaturées en espèces (BELKESSAM et al., 1997), ce qui explique le succès des 26 espèces de poissons introduites jusqu'à présent (KEITH et ALLARDI, 1997). Cette insaturation se retrouve aussi au niveau des fleuves côtiers et des îles (BELKESSAM et al., 1997). Mais ceci indique aussi la possibilité de naturalisation d'autres espèces, thème de ce séminaire.

\section{Niche écologique et compétition}

Dans les deux cas de l'acclimatation et de la naturalisation, le succès de l'introduction dépend de la niche écologique de l'espèce introduite, c'est-à-dire des ressources trophiques qu'elle exploite, mais aussi de son habitat au sens large : substrat, lieux de ponte éventuelle, abri des jeunes stades puis des adultes, milieux (ou hôtes) intermédiaires entre ces lieux ou abris. Si la niche et l'habitat d'introduction sont vides [collections d'eaux froides déjà mentionnées, niche des planctonophages de nombreux lacs tropicaux (LEVEQUE, 1997), niche pélagique des réservoirs nouvellement créés sur des cours d'eau rapides, et que l'ichthyofaune rhéophile ne peut coloniser], il n'y aura pas compétition et le succès de l'introduction dépendra des capacités d'adaptation de l'espèce introduite aux conditions physico-chimiques du milieu et de ses capacités d'insertion dans les réseaux trophiques existants (proies et prédateurs). Par contre, dans le cas où la niche ou l'habitat sont déjà occupés, il y aura compétition si la ressource commune n'est disponible qu'en quantité limitée pour chaque individu - cette ressource en vient donc à déterminer la biomasse des espèces qui l'exploitent. Par exemple, à l'air libre, il n'y a pas compétition entre les animaux pour l'oxygène atmosphérique; par contre, la surface des substrats durs limite l'effectif des moules susceptibles de s'y fixer, les zones de graviers propices à la ponte (frayères) limitent souvent l'effectif des populations de salmonidés (DELACOSTE et al., 1997). Si, dans la compétition, il y a superposition complète entre les niches de l'espèce introduite et d'une espèce préexistante, l'une des deux disparaîtra en application du principe d'exclusion compétitive de GAUSE, selon lequel deux compétiteurs absolus ne peuvent pas coexister. Seul subsistera celui qui se montrera le plus performant dans les conditions présentes du milieu. C'est l'un des mécanismes de l'élimination, soit de l'espèce introduite, soit de l'espèce indigène. Le corollaire du même principe s'énonce " la différenciation écologique est la condition nécessaire de la coexistence " (HARDIN, 1960) : pour que l'espèce introduite coexiste avec les espèces indigènes, il faut une différence dans leurs niches écologiques. On saisit l'importance majeure de ce concept. Le hotu, plus récemment arrivé, ne peut être accusé de compétition avec des espèces indigènes plus " nobles " (ombre commun) : végétarien, il est pratiquement seul à racler la couverture biologique du sédiment. II coexiste donc avec la plupart des espèces indigènes, sans leur nuire (NELVA, 1997). Par contre, les salmonidés planctonophages tendent à s'exclure l'un l'autre, dans les lacs pauvres d'altitude. Toutefois, dans quelques cas, des changements de microhabitats ont pu être observés chez d'autres espèces (BROWN et MOYLE, 1991 ; CRIVELLI, 1995). Outre cette compétition alimentaire et spatiale " indirecte ", existe également une compétition dite " directe " et liée au comportement : HELAND et BEALL (1997) ont montré que, 
par sa date d'éclosion, son comportement de nage et d'alimentation, le saumon coho domine le saumon atlantique, en limitant son accès aux ressources alimentaires et en le cantonnant dans les radiers; ce qui illustre le phénomène de ségrégation compétitive.

\section{Prédation et parasitisme}

La prédation et le parasitisme sont réglés par le cycle cybernétique prédateur-proie : le prédateur limite le nombre de ses proies en les consommant et, en rétroaction négative, le nombre des proies limite le nombre des prédateurs par la disponibilité alimentaire. A l'extrême, il y a risque d'extinction aussi bien du prédateur (ou du parasite) que de la proie (ou de l'hôte). Lorsque l'un et l'autre ont la possibilité de vivre ensemble un certain temps, il se produit une co-évolution, avec sélection entre les souches de prédateurs ou parasites et celles de la proie ou de l'hôte. Quelles seront les souches du prédateur qui auront le plus de chances de survivre et se perpétuer ? Sauf exception (LAMBERT, 1997), celles qui épargnent leurs proies (la poule aux œufs d'or !), donc les moins performantes en prédation - et celles-ci seront favorisées par sélection naturelle. II en va de même pour le parasitisme.

Par voie de conséquence, il est possible d'expliquer les explosions démographiques après introduction d'un prédateur ou d'un parasite inadapté, c'est-à-dire qui n'a pas subi la longue coévolution mentionnée par LAMBERT (1997) ; c'est le cas possible de Lates niloticus dans le lac Victoria (LEVEQUE, 1997 ; LOWE-McCONNEL, 1967, in GEE, 1991) et d'Anguillicola en Corse (ROCHE et MATTEl, 1997). Trop performante ou dépourvue de prédateur, l'espèce introduite trouve des ressources abondantes sans être elle-même contrôlée ; ses effectifs explosent. Mais, de ce fait, elle consomme ou utilise, et en vient à raréfier ces ressources; la prolifération est souvent suivie d'un effondrement des effectifs du consommateur (courbe en J), qui remontent de nouveau lorsque la ressource s'est reconstituée. On a ainsi des oscillations d'effectifs et de biomasse, avec mauvaise exploitation des ressources du milieu. Parfois, le cannibalisme permet à un prédateur introduit de réguler, au moins partiellement, l'effectif de ses populations. II faut noter qu'un prédateur ou un parasite devient aussi plus performant dans un milieu artificiellement simplifié (MORETEAU, communication orale) (monoculture, élevage intensif, où la densité des proies ou des hôtes est grande), et que les risques de pullulation de nuisibles sont particulièrement sévères dans de tels milieux. Une synergie entre la dégradation des milieux (eutrophisation, artificialisation) et les impacts des populations introduites semble aggraver la régression souvent constatée des espèces autochtones (comme pour les écrevisses, LAURENT, 1997 ; VIGNEUX, 1997).

\section{Insertion dans les réseaux trophiques}

Les niveaux trophiques se contrôlent assez généralement deux à deux. Les herbivores limitent la végétation, les prédateurs limitent les herbivores, les super prédateurs ou les parasites limitent les prédateurs, etc. Renforcer par une introduction les prédateurs que sont les poissons consommateurs de zooplancton revient à restreindre l'effet des herbivores et donc à favoriser les végétaux (avec les risques d'eutrophisation ou ses conséquences sur les proliférations phytoplanctoniques que l'on connaît), mais introduire un carnassier (super-prédateur) limite les effets précédents, en favorisant le zooplancton, lui-même susceptible de réduire la prolifération des algues ; ces super-prédateurs sont alors des espèces-clés de l'édifice trophique (PAINE, 1966, in BARBAULT, 1992). De même, on attribue à l'introduction du sandre en Turquie, la réduction des peuplements de cyprinidés et le développement des peuplements d'écrevisses, dont les cyprinidés mangeaient les larves (COWX, 1997).

La position dans les réseaux trophiques est donc majeure (SCHINDLER, 1991) mais, d'une part, il s'agit non pas de chaînes simples mais de réseaux dans lesquels la même espèce peut occuper des niveaux différents et, d'autre part, en raison de la méconnaissance de l'adaptation potentielle à des proies inconnues, la prospective d'insertion devient souvent une prévision simpliste, ce qui est amplement démontré par l'exemple de Mysis relicta. Sur ce point, la prudence de ROSECCHI et al. (1997) quant aux impacts des populations introduites sur les populations autochtones est à préconiser. 


\section{CONCLUSIONS : QUELQUES RECOMMANDATIONS D'ENSEMBLE}

\section{Préliminaires à une introduction volontaire}

Plusieurs questions doivent être posées d'emblée. De la réponse qui leur est apportée dérive la démarche d'introduction.

Quel sera le critère d'une "bonne " introduction ? Une production de biomasse maximale ? Une production à valeur marchande maximale? Une biodiversité spécifique (ou génétique) maximale ? La sauvegarde des espèces locales, à savoir le maintien de la diversité et de la production actuelle - en sachant, comme indiqué en introduction, qu'elles sont provisoires à l'échelle géologique, voire même historique?

Quelle est la définition d'une nuisance dans l'absolu ? Nuisance pour quelle activité ? Par exemple, l'introduction de poissons carnassiers est interdite dans les eaux de première catégorie piscicole. Dans ce cas, le critère est d'éviter une concurrence à la truite, donc un critère économique et lié à sa pêche sportive.

Le premier point consiste donc à trouver un accord sur la politique à mener, à la lumière des diverses prospections socio-économiques et écologiques. La recherche écologique doit ensuite étayer la législation, pour fournir les moyens de cette politique. Vu la perméabilité mentionnée des barrières terrestres de faible largeur, toute autorisation d'importer une espèce exotique à des fins de culture ou d'élevage implique à plus ou moins brève échéance son introduction dans les milieux naturels, et donc son acclimatation ou sa naturalisation si elle est capable de s'y maintenir. Avant d'énoncer un règlement, il convient toujours de prévoir les moyens pour assurer son application. Se retrancher derrière un règlement inapplicable tend à démobiliser les services responsables et constitue une démarche plus nuisible qu'utile. Des campagnes d'information et de sensibilisation du public sont indispensables.

Trois questions méritent des études :

1/ Est-il souhaitable d'introduire telle espèce?

2/ Faut-il se protéger contre telle espèce?

3/ Si oui, comment l'empêcher de gagner le territoire concerné ?

En dehors d'un certain nombre de réponses de bon sens liées aux règles énoncées aux chapitres 3 et 5 , une recommandation ne peut être faite qu'à la suite d'investigations scientifiques sur le fonctionnement actuel des écosystèmes aquatiques concernés. Le risque d'apparition d'une espèce donnée pourra être évalué d'après ses modalités de dispersion et ses capacités d'adaptation, comme il a été fait pour Corbicula dans un canal du Sud-Ouest de la France (DUBOIS, communication orale). Les risques de sa naturalisation pourront être évalués par le degré d'occupation de la niche écologique et la performance dans cette niche de l'espèce en cause. C'est donc l'ensemble du groupe fonctionnel dans lequel l'espèce introduite doit s'insérer qu'il faudra étudier. Cette stratégie scientifique devrait notamment s'appliquer dans le cas de parasites dont on souhaite protéger les organismes indigènes. L'étude sur le nématode parasite Anguillicola (survie du stade libre, hôtes intermédiaires potentiels, temps de génération et possibilité de dispersion) en constitue un exemple démonstratif (ASHWORTH et BLANC, 1997). Toutefois, il est souvent difficile de prévoir la nuisibilité d'espèces introduites hors de leur milieu d'origine, car les compétitions et régulations existantes se trouvent absentes du nouveau milieu colonisé : les introductions en milieu naturel devraient être réalisées d'abord en eaux closes, et suite à des études expérimentales. Une grille d'analyse des problèmes en cas d'introduction volontaire pourrait alors contribuer à la prise de décision (figure 2).

\section{Se prémunir des introductions involontaires ou les gérer?}

II faut cesser de considérer la France comme un continent à part. Comme le montrent de nombreux exemples étrangers (DUTARTRE et HAURY, communication orale), toute politique d'introduction devrait se pratiquer à l'échelle européenne, étant donné la perméabilité biologique évidente de nos frontières, au moins en plaine ainsi qu'au niveau des ports et aéroports. A plus 


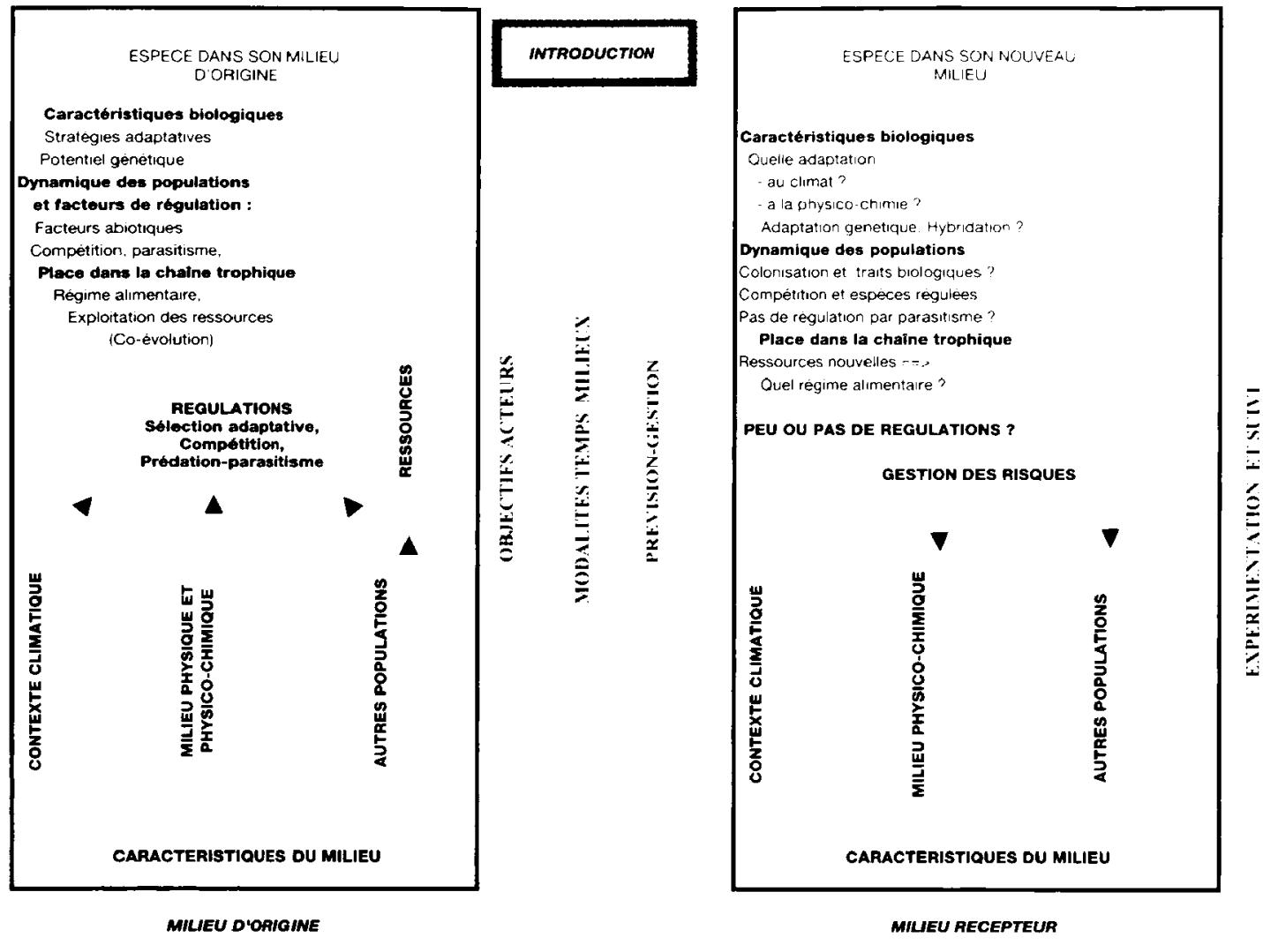

PRINCIPE DE PRECAUTION

AISQUE
D'EVASION

Figure 2

Proposition de grille d'analyse préliminaire à une introduction volontaire d'espèce.

Figure 2

Proposal for a general investigation before introducing any species.

forte raison, les listes départementales d'espèces présentes ou à ne pas introduire sont inadéquates. La seule base objective pour de telles listes est le bassin fluvial, comme le prévoit la Loi sur l'Eau (GALLAND, 1997), tout en conservant à l'esprit sa liaison avec d'autres bassins par les canaux, si bien que le suivi de l'information étrangère à chaque bassin et à chaque État européen est maintenant devenu une nécessité.

Partant de la constatation que toute espèce introduite, même en eaux closes ou en milieu confiné, présente un risque d'acclimatation, puis de naturalisation et d'expansion, il est nécessaire d'envisager d'emblée la gestion des risques et d'appliquer un principe de précaution. Les participants à ce colloque ont souligné la très grande difficulté de la gestion après introduction. Ceci amène à préconiser une surveillance autour des sites privilégiés d'introduction, en fonction des espèces susceptibles de gagner notre territoire. De ce fait, il y a nécessité de maintenir des biologistes sur le terrain, voire d'en accroitre le nombre. Il s'agira pour eux d'assurer une "veille écologique " (à l'instar de ce qui se pratique en protection des cultures), afin de pouvoir répondre aux gestionnaires dans des délais compatibles avec leurs besoins et éviter que des invasions dommageables viennent s'ajouter aux autres atteintes actuelles des systèmes aquatiques. II est parfois possible, sinon d'éradiquer les espèces introduites (castor américain dans l'Oise), tout au moins de maintenir les populations d'espèces introduites en-deçà d'un seuil 
de nuisibilité ; ce qui est actuellement l'objet du contrôle des plantes aquatiques. Par ailleurs, comme mentionné auparavant, des stabilisations de populations peuvent intervenir naturellement. L'homme peut alors contribuer à accélérer ce processus de régulation.

Enfin, les exemples d'information du public réalisés à l'étranger pour les espèces riveraines de cours d'eau (ANONYME, 1994) ou en France sur la tortue de Floride (MINISTĖRE DE L'ENVIRONNEMENT, 1995) devraient permettre de limiter les introductions malencontreuses ou les lâchers abusifs.

Quant à la question du gain de biodiversité à attendre des introductions d'espèces, il semble bien que le bilan soit assez largement négatif, et que toute introduction corresponde souvent à une mise en danger des espèces autochtones et à une artificialisation accrue des systèmes. L'argument d'enrichissement spécifique devrait donc s'effacer devant le principe de précaution, tant que les études scientifiques n'auront pas montré l'innocuité de telle ou telle introduction.

Il est néanmoins patent que peu d'études écosystémiques existent en France sur les effets des introductions dans les hydrosystèmes, portant notamment sur les différents compartiments abiotiques et biotiques ainsi que sur les réseaux trophiques. Avant de pouvoir parler de déséquilibres écologiques induits par des introductions, il reste donc nécessaire de mieux décrypter les domaines de variabilité naturelle des hydrosystèmes non perturbés.

\section{BIBLIOGRAPHIE}

ALLAN J.D., 1995. Stream Ecology - Structure and function of running waters. Chapman and Hall, London, $388 \mathrm{p}$.

AMOROS C., PETTS G.E., 1993. Hydrosystèmes fluviaux. Masson, Paris, 300 p.

ANONYME, 1994. Guidance for the control of invasive plants near watercourses. National River Authorities, $21 \mathrm{p}$.

ARTHINGTON A.H., MITCHELL D.S., 1986. Aquatic invading species. In GROVES R.H. and BURDON J.J. (Eds.), Ecology of biological invasions, 34-53. Cambridge University Press, Cambridge.

ASHWORTH S.T., BLANC G., 1997. Anguillicola crassus, un colonisateur agressif récemment introduit dans les stocks européens d'anguilles. Bull. Fr. Pêche Piscic., 344-345.

BACHMANN V., USSEGLIO-POLATERA P., CEGIELKA E., WAGNER P., POINSAINT J.F., MORETEAU J.C., 1997. Premières observations sur la coexistence de Dreissena polymorpha, Corophium curvispinum et Corbicula spp. dans la rivière Moselle. Bull. Fr. Pêche Piscic., 344-345.

BAGLINIERE J.L., THIBAULT M., DUMAS J., 1990. Réintroductions et soutiens de populations du saumon atlantique (Salmo salar L.) en France. Rev. Ecol. (Terre Vie), Suppl. 5, 299-323.

BARBAULT R., 1992. Écologie des peuplements - Structure, dynamique et évolution. Masson, Paris, $273 p$.

BARRETT S.C.H., RICHARDSON B.J., 1986. Genetic attributes of invading species. In GROVES R.H. and BURDON J.J. (Eds.), Ecology of biological invasions, 21-33. Cambridge University Press, Cambridge.

BELKESSAM D., OBERDORFF T., HUGUENY B., 1997. Unsaturated fish assemblages in rivers of the North Western France : potential consequences for species introductions. Bull. Fr. Pêche Piscic., 344-345.

BENTATA V., 1997. Le statut juridique en droit interne des espèces animales liées aux milieux aquatiques continentaux métropolitains. Bull. Fr. Pêche Piscic., 344-345. 
BERREBI P., 1997. Introduction d'espèces dans les milieux aquatiques d'eau douce : les impacts génétiques. Bull. Fr. Pêche Piscic., 344-345.

BLANC G., 1997. L'introduction des agents pathogènes dans les écosystèmes aquatiques : aspects théoriques et réalitès. Bull. Fr. Pêche Piscic., 344-345.

BROWN L.R., MOYLE P.B., 1991. Changes in habitat and microhabitat partitioning within an assemblage of stream fishes in response to predation by Sacramento squawfish (Ptychocheilus grandis). Can. J. Fish. Aquat. Sci., 48, 849-856.

BURKE M.J.W., GRIME J.P., 1996. An experimental study of plant community invasibility. Ecology, 77, 776-790.

CARY P.R., WEERTS P.G.K., 1983. Growth of Salvinia molesta as affected by water temperature and nutrition. I. Effects of nitrogen level and nitrogen compounds. Aquat. Bot., 16, 163172.

COOK C.D.K., 1985. Range extensions of aquatic vascular species. J. Aquat. Plant Manage., 23, $1-6$.

COSTE M., 1994. Sur l'utilisation des diatomées dans le diagnostic biologique de la qualité des eaux courantes - Intérêt et limite des indices. In CHARTIER-TOUZE N., COLLIN M.P., GALVIN Y., Les variables biologiques de la qualité des écosystèmes aquatiques, Min. Environn., GIP Hydrosystèmes, A.G.H.T.M., Paris, 2-3 nov. 1994, 135-152.

COWX I.G., 1997. L'introduction d'espèces de poissons dans les eaux douces européennes : succès économiques ou désastres écologiques ? Bull. Fr. Pêche Piscic., 344-345.

CRIVELLI A.J., 1995. Are fish introductions a threat to endemic freshwater fishes in the Northern Mediterranean Region? Biol. Conserv., 72, 311-319.

DAVAINE P., BEALL E., 1997. Introduction de salmonidés en milieu vierge (îles Kerguelen, Subantarctique) : enjeux, résultats, perspectives. Bull. Fr. Pêche Piscic., 344-345.

DELACOSTE M., BARAN P., LASCAUX J.M., ABAD N., BESSON J.P., 1997. Bilan des introductions de salmonidés dans les lacs et ruisseaux d'altitude des Hautes-Pyrénées. Bull. Fr. Pêche Piscic., 344-345.

DUMAS J., HAURY J., 1995. Une rivière du Piémont pyrénéen : la Nivelle (Pays basque). Acta Biologica Montana, 11, 113-146.

DUTARTRE A., HAURY J., PLANTY-TABACCHI A.M., 1997. Introductions de macrophytes aquatiques et riverains dans les hydrosystèmes français métropolitains : essai de bilan. Bull. Fr. Pêche Piscic., 344-345.

ELVIRA B., 1995. Native and exotic freshwater fishes in Spanish river basins. Freshwat. Biol., 33, 103-108.

GALLAND J.P., 1997. Les nouvelles dispositions du Code rural sur les introductions d'espèces issues de la loi du 2 février 1995 relative au renforcement de la protection de l'environnement. Bull. Fr. Pêche Piscic., 344-345.

GEE J.H.R., 1991. 9 - Speciation and biogeography. In BARNES R.S.K., MANN K.H., Fundamentals of Aquatic Ecology, 2nd Ed., 172-185, Blackwell Sci. Publ., Oxford.

GROVES R.H., BURDON J.J. (Eds.), 1986. Ecology of biological invasions. Cambridge University Press, Cambridge, $166 \mathrm{p}$.

GUEVEL B., 1997. La "Loi Pêche " (Code rural) et l'introduction des espèces piscicoles. Bull. Fr. Pêche Piscic., 344-345.

HARDIN G., 1960. The competitive exclusion principle. Science, 131, 1292-1297. 
HELAND M., BEALL E., 1997. Etude expérimentale de la compétition interspécifique entre juvéniles de saumon coho, Oncorhynchus kisutch, et de saumon atlantique, Salmo salar, en eau douce. Bull. Fr. Pêche Piscic., 344-345.

HUTCHINSON G.E., 1975. A treatise on limnology. III. Limnological botany. J. Wiley and Sons, New York, $660 \mathrm{p}$.

KAUFMAN L., 1992. Catastrophic change in species-rich freshwater ecosystems. The lessons of Lake Victoria. Bioscience, 42, 846-858.

KEITH P., ALLARDI J., 1997. Bilan des introductions de poissons d'eau douce en France. Bull. Fr. Pêche Piscic., 344-345.

KHALANSKI M., 1997. Conséquences industrielles et écologiques de l'introduction de nouvelles espèces dans les hydrosystèmes continentaux : la moule zébrée et autres espèces invasives. Bull. Fr. Pêche Piscic., 344-345.

LAMBERT A., 1997. Introduction de poissons dans les milieux aquatiques continentaux : "Quid de leurs parasites ? ». Bull. Fr. Pêche Piscic., 344-345.

LAURENT P.J., 1997. Introductions d'écrevisses en France et dans le monde, historique et conséquences. Bull. Fr. Pêche Piscic., 344-345.

LEVEQUE C., 1994. Etat de santé des écosystèmes aquatiques: I'intérêt des variables biologiques. In CHARTIER-TOUZE N., COLLIN M.P., GALVIN Y. (Eds.), Les variables biologiques de la qualité des écosystèmes aquatiques, Min. Environn., GIP Hydrosystèmes, A.G.H.T.M., Paris, 2-3 nov. 1994, 12-24.

LEVEQUE C., 1997. Introductions de nouvelles espèces de poissons dans les eaux douces tropicales : objectifs et conséquences. Bull. Fr. Pêche Piscic., 344-345.

MACARTHUR R.H., WILSON E.O., 1967. The theory of island biogeography. Princeton University Press, Princeton, New Jersey, 203 p.

MATTHEY G., 1966. Deux espèces nouvelles de la faune du Léman : Dreissena polymorpha Pallas (Mollusca, Dreissenidae), Acerina cernua (L.) (Pisces, Percidae). Bull. Soc. vaud. Sci. nat., 69, 229-232.

MAURIN H., 1997. L'homme et les mammifères de France métropolitaine : évolution historique et introductions d'espèces dans les milieux humides et aquatiques. Bull. Fr. Pêche Piscic., 344-345.

MINISTĖRE DE L'ENVIRONNEMENT, 1995. Les tortues de Floride, animaux familiers. Les connaître pour en prendre soin. PRODAF-Ministère Environnement, Paris, $24 \mathrm{p}$.

MOUTOU F., 1997. Mammifères aquatiques et semi-aquatiques introduits en France. Risques et conséquences. Bull. Fr. Pêche Piscic., 344-345.

MURRAY N.D., TOMASOV J.F., HALE C.B., 1986. Ecological and genetic differentiation between Australian populations of introduced biological control agents. In GROVES R.H. and BURDON J.J., Ecology of biological invasions : 154. Cambridge University Press, Cambridge.

NELVA A., 1997. La pénétration du Hotu, Chondrostoma nasus nasus (Poisson Cyprinidé), dans le réseau hydrographique français et ses conséquences. Bull. Fr. Pêche Piscic., 344-345.

NEVEU A., 1997. L'introduction d'espèces allochtones de grenouilles vertes en France, deux problèmes différents : celui de $R$. Catesbeiana et celui des taxons non présents du complexe Esculenta. Bull. Fr. Pêche Piscic., 344-345.

NEWSOME A.E., NOBLE I.R., 1986. Ecological and physiological characters of invading species. In GROVES R.H., BURDON J.J., Ecology of biological invasions : 1-20. Cambridge University Press, Cambridge. 
NORTHCOTE T.G., 1972. Some effects of mysid introduction and nutrient enrichment on an oligotrophic lake and its salmonids. Verh. Internat. Verein. Limnol., 18, 1096-1106.

PERENNOU C., 1997. L'Erismature rousse (Oxyura jamaicensis) : une introduction problématique d'oiseau dans les milieux aquatiques. Bull. Fr. Pêche Piscic., 344-345.

PERSAT H., KEITH P., 1997. La répartition géographique des poissons d'eau douce en France : qui est autochtone et qui ne l'est pas? Bull. Fr. Pêche Piscic., 344-345.

PLANTY-TABACCHI A.M., 1997. Invasions des corridors fluviaux du Sud-Ouest par des espèces végétales exotiques. Bull. Fr. Pêche Piscic., 344-345.

POURRIOT R., MEYBECK M., 1995. Limnologie générale. Masson, Paris, $956 \mathrm{p}$.

RICHARDS R.C., GOLDMAN C.R., FRANTZ T.C., WICKWIRE R., 1975. Where are all the Daphnia gone? The decline of a major cladoceran in Lake Tahoe, California-Nevada. Verh. Internat. Verein. Limnol., 19, 835-842.

ROCHE B., MATTEI J., 1997. Les espèces animales introduites dans les eaux douces de Corse. Bull. Fr. Pêche Piscic., 344-345.

ROSECCHI E., POIZAT G., CRIVELLI A.J., 1997. Introductions de poissons d'eau douce et d'écrevisses en Camargue : historique, origines et modifications des peuplements. Bull. Fr. Pêche Piscic., 344-345.

ROSS S.T., 1991. Mechanisms structuring stream fish assemblages : are there lessons from introduced species ? Environ. Biol. Fish., 30, 359-368.

SASTRE C., 1997. Les introductions d'espèces dans le milieu aquatique. Le contexte biogéographique : aspects généraux et paléohistoire. Bull. Fr. Pêche Piscic., 344-345.

SASTRE C., BENTATA V., 1997. Propositions de définitions pour les termes biogéographiques utilisés. Bull. Fr. Pêche Piscic., 344-345.

SCHINDLER D.W., 1991. 6 - Aquatic ecosystems and global ecology. In BARNES R.S.K., MANN K.H., Fundamentals of aquatic ecology, 2nd Ed., 108-122, Blackwell Sci. Publ., Oxford.

SERVAN J., ARVY C., 1997. Introduction de la tortue de Floride Trachemys scripta en France. Un nouveau compétiteur pour les espèces de tortues d'eau douce européennes. Bull. Fr. Pêche Piscic., 344-345.

SPENCER C.N., McCLELLAND B.R., STANFORD J.A., 1991. Shrimp stocking, salmon collapse and eagle displacement. BioScience, 41, 14-21.

SYTSMA M.D., ANDERSON L.J.W., 1993. Criteria for assessing nitrogen and phosphorus deficiency in Myriophyllum aquaticum. J. Freshw. Ecol., 8, 155-163.

THIEBAUT G., ROLLAND T., ROBACH F., TREMOLIERES M., MULLER S., 1997. Quelques conséquences de l'introduction de deux espèces de macrophytes, Elodea canadensis Michaux et Elodea nuttallii St. John, dans les écosystèmes aquatiques continentaux: exemple de la plaine d'Alsace et des Vosges du Nord (Nord-Est de la France). Bull. Fr. Pêche Piscic., 344-345.

VIGNEUX E., 1997. Les introductions de crustacés décapodes d'eau douce en France, peut-on parler de gestion? Bull. Fr. Pêche Piscic., 344-345. 